\title{
Dynamic characterization of tungsten carbide behaviour at very high strain-rates
}

\author{
Benjamin Erzar ${ }^{1, *}$, Jean-Luc Zinszner ${ }^{1}$ \\ ${ }^{1}$ CEA, DAM, GRAMAT, BP 80200, F-46500 Gramat, France
}

\begin{abstract}
Cemented tungsten carbide, with its very high density and high strength, is known to be the material composing several small calibre armour piercing ammunitions. The impact of a tungsten carbide core projectile onto a high efficiency armour often leads to the fracture of the tungsten carbide core. Thus, the pertinence of material models used in numerical simulations to describe the behaviour and the damage of the target is not sufficient to well predict a ballistic impact. In this work, the GEPI high-pulsed power generator is used to conduct dynamic characterization of the behaviour of a cemented tungsten carbide under both compressive and tensile loadings. The Hugoniot Elastic Limit of this material has been identified $(\mathrm{HEL}=5.8 \mathrm{GPa})$. Moreover, Lagrangian analysis allowed the complete loading path to be identified up to 18 GPa.
\end{abstract}

\section{Introduction}

With its very high density and high strength, cemented tungsten carbide is known to be the material composing several small calibre armour piercing ammunitions. The impact of an armour piercing projectile against a high efficiency armour (for example, multi-layered configuration composed of a ceramic material in the front face backed by a ductile plate) often leads to a fragmentation of both the ceramic but also of the projectile core. Thus, the improvement of the numerical models used to describe the dynamic response of the target is not sufficient to well reproduce, by numerical simulation, a ballistic impact. In order to be able to simulate the response of an armour configuration against a given threat, one needs to improve the numerical description of the plasticity, damage and fracture of perforating cores.

In order to characterize the dynamic compressive strength of materials and their plastic response, plate impact tests are generally performed. This kind of experiments allows the Hugoniot elastic limit (HEL) to be determined along with the Hugoniot curve which is the loci of all possible equilibrium states under shock loading. Moreover, the determination of yield strength of materials with the flyer plate technique requires intrusive techniques like lateral stress gauges embedded in the material. Several tests are necessary to determine accurately the shock response of such materials. In this work, dynamic compression experiments have been performed on a cemented tungsten carbide using a highpulsed power generator called GEPI. The GEPI machine is a low inductance electric generator, based on the stripline concept and composed of two electrodes separated by dielectric foils [1]. Using this generator, the same compressive pulse is applied on the two electrodes. Thus, by performing a compression experiment on two samples of a same material with different thicknesses, one can apply a Lagrangian analysis to reconstruct the whole loading path without any assumption.

First, a brief description of the microstructural characteristics of the cemented tungsten carbide characterized in this work is presented. The principles of the testing machine, the experimental method as well as a description of the Lagrangian analysis method are then summarized. In the third part, the experimental results are presented.

\section{Presentation of the cemented tungsten carbide}

The core of $7.62 \times 51$ AP8 projectiles is composed of a cemented tungsten carbide containing about $11 \% \mathrm{wt}$ of cobalt. This cobalt matrix has a huge influence on the behaviour of the material. The higher the cobalt content, the higher is the toughness of the cemented tungsten carbide. The hardness measured on the AP8 core is about $1365 \mathrm{HV}_{30}$. However, due to the small diameter of these cores, one cannot directly use these specimens to perform dynamic tests such as plate impact experiments or GEPI dynamic tests. An equivalent material, called h40 (12 wt\% Co) and provided by Carbure $d u$ Chéran (France), has been used. It presents similar microstructural characteristics in terms of grain size and binder content in comparison of the AP8 core material. It also presents a similar a hardness value (1395 $\mathrm{HV}_{30}$ ). Figure 1 presents SEM pictures of the AP8 core material (a) and of the equivalent tungsten carbide (h40 grade) (b).

Corresponding author: benjamin.erzar@cea.fr 


\section{Principle of isentropic compression experiments}

\subsection{Principle of GEPI device}

The GEPI machine is based on High Pulsed Power (HPP) technologies.
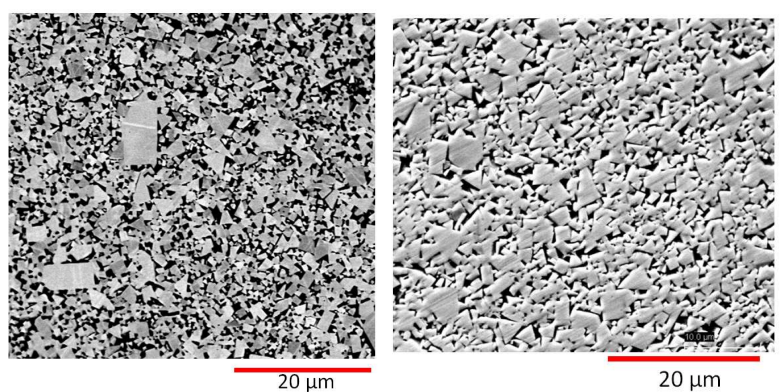

Fig. 1. SEM pictures of microstructures from AP8 core (a) and the equivalent tungsten carbide (h40 grade) (b).

This generator applies the strip line concept [1]: two aluminium plane electrodes, separated by dielectric foils and locally connected by a short circuit are subjected to an intense current focused on the load region. In those experiments, the current reaches a maximum intensity of 3.3 MA in nearly $0.5 \mu \mathrm{s}$. The association of current and magnetic field produces Lorentz forces applied on the internal face of the electrodes. The pressure pulse applied to the electrode can be directly computed (cf. Eq. 1) knowing the evolution of the current with time $I(t)$, the width of the terminal part of the electrode $W$, the vacuum permeability $\mu_{0}$ and an edge effect factor $\mathrm{kp}_{\mathrm{p}}$ (near 1$)$.

$$
P_{\text {mag }}(t)=\frac{1}{2} k_{p} \mu_{0}\left[\frac{I(t)}{W}\right]^{2}
$$

This machine is generally devoted to isentropic compression experiments thanks to the ramp loading. During experiments, the compressive pulse propagates through the electrodes and the specimens before reaching $\mathrm{LiF}$ windows.

\subsection{Experimental configuration}

Due to the symmetry of the GEPI configuration (Figure 2), two specimens can be tested during one GEPI compression test. This configuration also allows using the Lagrangian analysis method when a GEPI compression test is performed on two specimens of a same material but having different thicknesses.

In this work, two GEPI compression test are performed on the h40 cemented tungsten carbide. The thicknesses of the specimens are $3.5 \mathrm{~mm}$ and $4.5 \mathrm{~mm}$.

The electrodes' widths are set to 25 and $20 \mathrm{~mm}$. These widths allow ensuring a compressive pulse sufficiently high (about 10 and $18 \mathrm{GPa}$ respectively) in order to exceed the HEL of the material. The specimens' widths are chosen in order to ensure that the interface velocities measured on the two specimens are not affected by radial release waves during the compressive loading.

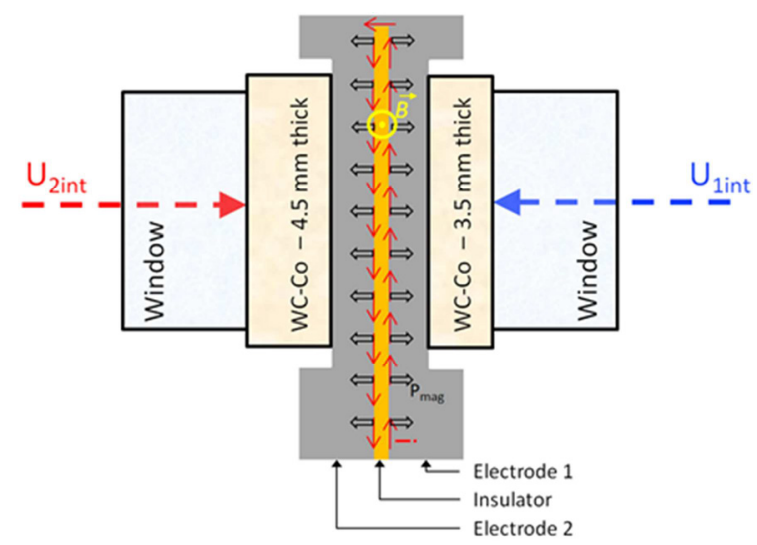

Fig. 2. Experimental configuration used to perform GEPI compression tests and allowing using Lagrangian analysis method.

\subsection{Lagrangian analysis method}

The Lagrangian analysis method realised after one GEPI compression test allows determining the whole loading path of the material whereas only one point on this loading path can be obtained after one plate impact experiment. This method is based on the integration of the equations of conservation of mass, momentum and energy [2]. Moreover, this method does not need any assumption on the behaviour of the material. The principle is based on a comparison of velocity signals obtained on two different thicknesses of a material.

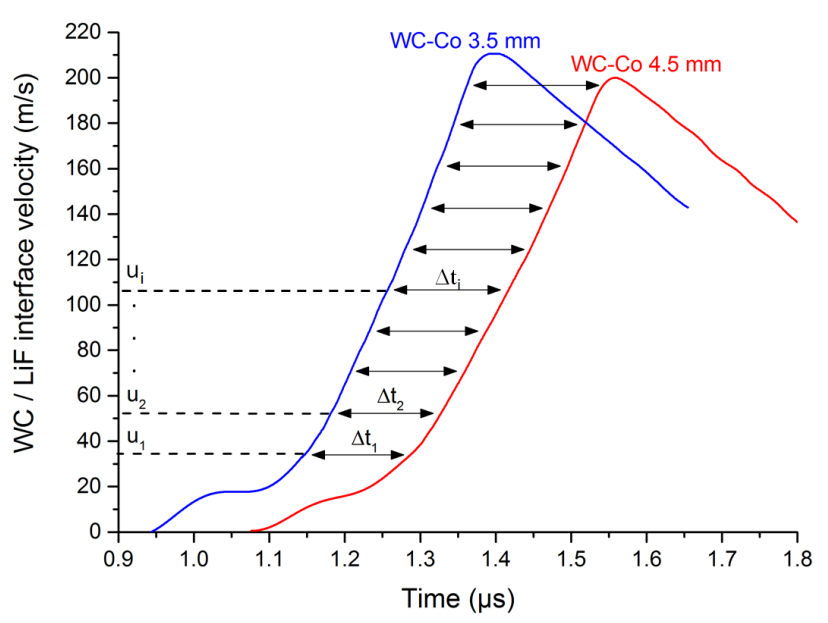

Fig. 3. Principle of Lagrangian analysis method: determination of the wave velocity $C_{L}$ associated to each particle velocity $u_{i}$ (arbitrary origin of time).

For each particle velocity $u_{i}$, an associated wave velocity $C_{L}\left(u_{i}\right)$ can be calculated from travel times $\Delta \mathrm{t}_{\mathrm{i}}$ (Figure 3 ). Then, this wave velocity is used to integrate increments of longitudinal stress $\sigma_{x}$, longitudinal strain $\varepsilon_{x}$ (stress and strain are considered positives in compression) or specific volume associated to each increment of particular velocity following:

$$
d \sigma_{x}=\rho_{0} C_{L}\left(u_{i}\right) d u_{i}
$$




$$
\begin{gathered}
d v=-\frac{1}{\rho_{0} C_{L}\left(u_{i}\right)} d u_{i} \\
d \varepsilon_{x}=\frac{d u_{i}}{C_{L}\left(u_{i}\right)}
\end{gathered}
$$

It can be noted that a correction based on the mechanical impedances of the ceramic and of the window material has to be realised to determine the in-situ particle velocity using the measured interface velocity.

\section{Experimental results}

Figure 3 presents the velocity signals measured during one of the two GEPI experiments. From these velocity profiles, a Lagrangian analysis has been performed. Figure 4 presents the results plotted in the stress/strain plane for the two GEPI tests. One can see that the two GEPI tests give very similar results showing the repeatability of these tests.

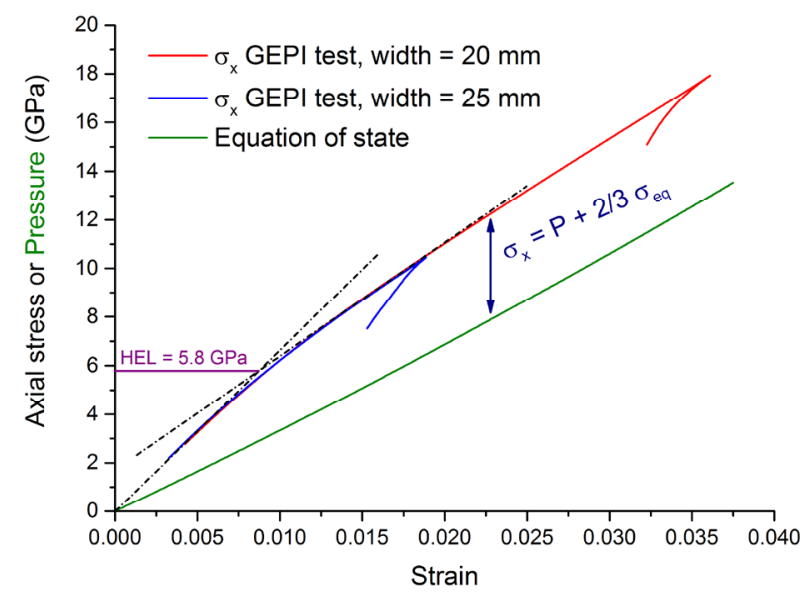

Fig. 4. $\mathrm{SiC} / \mathrm{LiF}$ interface velocity signals obtained on the two PS-S specimens after GEPI compression test (arbitrary origin of time).

The Hugoniot Elastic Limit (HEL) of tungsten carbide material is difficult to determine by using only the interface velocity signals. Indeed, the slope change in velocity signals generally observed when the HEL of a material is reached is not clearly observable (Figure 3). Nevertheless, the HEL of the h40 cemented tungsten carbide can be easily determined from Lagrangian analysis results (Figure 4). By plotting the stress/strain curve, the elastic/inelastic transition is observed at about $5.8 \mathrm{GPa}$. Despite its higher cobalt content, this Hugoniot Elastic Limit is close to then one obtained by Grady for Cercom tungsten carbide (high purity tungsten carbide) [3].

The longitudinal stress obtained with the Lagrangian analysis method is not sufficient to determine the equivalent stress. However, during a uniaxial deformation or for all triaxial loading such as $\sigma_{y}=\sigma_{z}$, one can write:

$$
\sigma_{e q}=\frac{3}{2}\left(\sigma_{x}-P\right)
$$

with the axial stress $\sigma_{x}>0$ in compression. Thus, by setting an equation of state for the cemented tungsten carbide, one can evaluate the evolution of the equivalent stress.

The equation of state of the h40 WC-Co $(12 \mathrm{wt} \% \mathrm{Co})$ content has been determined basing on a mixture law proposed by Saurel et al. [4]. The Hugoniot data of quasi pure tungsten carbide is given by Dandekar and Grady [5] and those of cobalt metal are obtained in LASL Shock Hugoniot data [5]. The obtained Hugoniot data for the h40 WC-Co are gathered in Table 1.

Table 1. Hugoniot data of h40 cemented tungsten carbide.

\begin{tabular}{|c|c|c|c|c|}
\hline Material & $\begin{array}{c}\text { Density } \\
(\mathrm{kg} / \mathrm{m} 3)\end{array}$ & $\begin{array}{c}\mathrm{C}_{0} \\
(\mathrm{~m} / \mathrm{s})\end{array}$ & $\mathrm{s}$ & $\begin{array}{c}\text { Grüneisen } \\
\text { coefficient }\end{array}$ \\
\hline $\begin{array}{c}\mathrm{h} 40 \\
\mathrm{WC}-\mathrm{Co} \\
(12 \% \mathrm{wt})\end{array}$ & 14356 & 4756 & 1.38 & 1.26 \\
\hline
\end{tabular}

Figure 5 presents the evolution of the yield strength as a function of the pressure obtained from lagrangian analysis results.

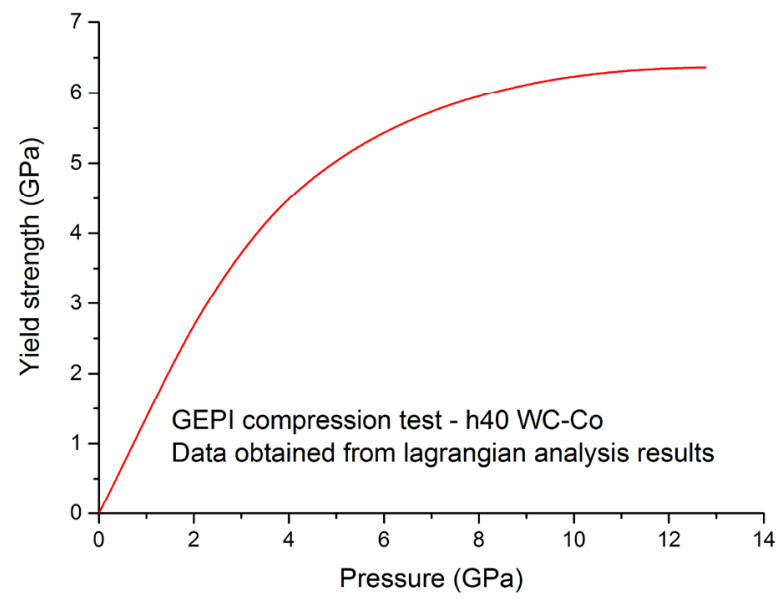

Fig. 5. Evolution of the yield strength of h40 tungsten carbide as a function of the pressure determined from GEPI compression tests.

\section{Conclusions}

In this work, dynamic compression experiments have been performed on a cemented tungsten carbide using a high pulsed power generator, called GEPI. This tungsten carbide grade, called $\mathrm{h} 40$, is representative of the 7.62 AP8 projectile core material. The use of the GEPI device allows realising a Lagrangian analysis with the velocity signals obtained and thus determining the whole loading path in the material. In a first time, the Hugoniot elastic limit (HEL) of the tungsten carbide has been determined. In a second time, the Lagrangian analysis allows the equivalent stress to be derived, providing useful data to determine material parameters to be used in numerical models for simulating the dynamic behaviour of armour piercing core. 


\section{References}

1. C. Mangeant, F. Lassalle, P. L'Eplattenier, P.L. Héreil, D. Bergues, G. Avrillaud, Shock compression of condensed matter, 1173 (2001)

2. J. Cagnoux, P. Chartagnac, P.L. Héreil, M. Perez, Ann. Phys. Fr. 12, 451 (1987)

3. D.E. Grady, US Army TARDEC report, Contract W56HZV-08-P-L594, ARA Project L18637, 2009
4. R. Saurel, O. Le Metayer, J. Massoni, S. Gavrilyuk, Shock Waves, 16, 209232 (2007)

5. D.P. Dandekar, D.E. Grady, Shock Compression of Condensed Matter, AIP (2001)

6. LASL Shock Hugoniot Data (Stanley P. Marsh editor, University of California Press, 1980) 